\title{
UAV 3D Mobility Model Based on Density Change Product
}

\author{
Rui Wang ${ }^{1,2, *}$, Nan $\mathrm{Di}^{3}, \mathrm{Na} \mathrm{Wang}^{1}$, Fangxin Liu ${ }^{1}$ and Qiuli Chen ${ }^{1}$ \\ ${ }^{1}$ PLA University of Science and Technology, China \\ ${ }^{2}$ NRIEE, China \\ ${ }^{3}$ Institute of China Electronic System Engineering Company, China \\ ${ }^{*}$ Corresponding author
}

\begin{abstract}
The network performance of UAV airborne is closely relied on the movement of the mobile nodes which is decided by the mobility mode. And the selection of mobility model and its parameters is of great significance to the simulation results. Based on the three-dimensional Gauss Markova mobility model (3D-GMM), this paper expand GM by using visit density and the changeable trend, and propose a new three-dimensional Gauss Markova mobility model based on density change product (3DDGMM). The results show that 3D_DGMM can simulate the movement of UAVs well and have higher event capture rate compared to 3D-GMM.
\end{abstract}

Keywords-component; airborne; node mobility; visit density; density change product; GMM

\section{INTRODUCTION}

Wireless ad hoc network has self-flexibility, dynamic coverage, and high robustness, which makes it widely applied in many fields [1]. Especially, combined with the development of data link in recent years, the airborne network based on wireless ad hoc network has played an important role in the field of aviation communication. The unmanned aerial vehicle (UAV) as the main body of UAV airborne network not only has the characteristics of traditional wireless ad hoc networks, such as dynamic topology, multi hop communication, distributed architecture and so on, but also has many other advantages, such as maneuvering speed, coverage is large in scale and the node energy [2]. This makes the UAV airborne network play a crucial role in monitoring air target tracking, space situation perception and interaction, no human machine automatic anti-collision, air land and sea collaborative operations and emergency rescue and other fields [3].

A reliable, efficient and real-time UAV airborne network must have a scientific and flexible mobility model, in order to cope with the challenges faced by the UAV airborne network, such as event situation capture, frequent link changes, complex environment and so on. Due to the particularity of high speed motion of UAV nodes, the traditional wireless ad hoc network mobility model can't be directly applied to the UAV airborne network [4]. To this end, Broyles designed and implemented the three-dimensional airborne network Gauss Markov mobile model (3D-GMM) based on the high dynamic performance of the airborne network in [5]. The model increases the pitch angle parameter for airborne nodes, enable nodes to be more flexible in the processing of aerial maneuvering. But due to the lack of consideration of the different motion state of the node, the model's simulation of the real movement of nodes in the air still exist a certain gap. Rohrer increased the effectiveness of the assessment on the basis of the 3D-GMM in [6], through emphasizing the adaptability of the node mobility and the diversity of the movement state. But the calculation is more complicated, and it is difficult to be applied in 3D space. Zhao proposed semi Markov smooth (SMS) mobility model based on 3D-GMM in [7], which studied acceleration condition, stability condition, deceleration condition and stationary condition of node motion, but the adaptability in airborne network is poor. Zheng proposed the three-dimensional Gauss smoothing semi Markov mobility model in [8], taking the actual environment noise and other factors into account, and increased the Gaussian disturbance in the model, but lacking the dealing with the node moving at boundary conditions. He inspired by fish and analyzed the way of transforming between nodes in different state, and designed 3D NMM (3D semi-random node mobility model) in [9], which achieved the effective coverage of regional monitoring target event, but the choice of the State adopts the way of random change, which lacks the analysis of the rationality of the choice of the next state.

This paper introduced visit density in the Gaussian model, improved the 3D-GMM model and proposed threedimensional Gauss Markova mobility model based on density change product (3D-DGMM). Thus the UAV physical movement more in line with reality, and improve the network performance.

\section{PROBLEM DESCRIPTION AND MODEL DEFINITION}

\section{A. Problem Description}

A simulated UAV network scene accurately analysis of wireless signal transmission, need to exactly know the location of the mobile node, and models of UAV mobile are directly related to the topology changes, impact the throughput of the whole network, transmission delay, routing efficiency index measurement, due to field test are not easy to control, dangerous, can't be reproduced and some other shortcomings and difficulties, for new applications and new protocol verification mainly through simulation technology, so it is necessary to have a UAV mobile model to simulate realistic UAV mobile. 
Integrating the advantages and disadvantages of existing mobile model, the model proposed in this paper has the following three characteristics: (1) close to realistic node mobility for smooth movement, speed and direction with correlation; (2) with controllable model parameters of strong, independent regulation; (3) node mobility model for hybrid mobile model, that is, influenced by time, space, geography. As shown in Figure I:

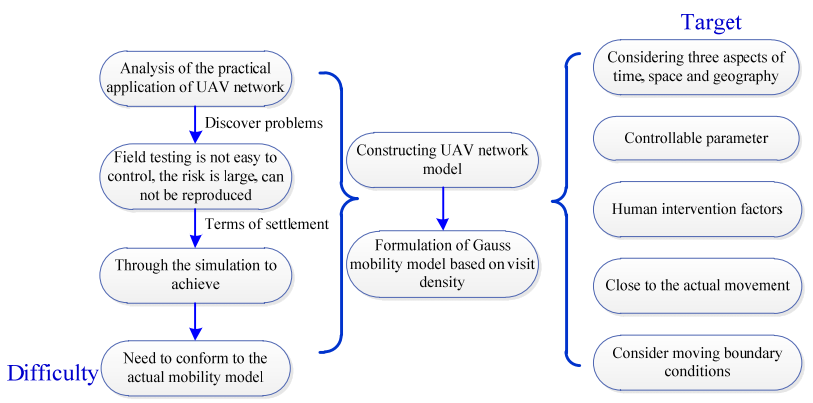

FIGURE I. THE CHARACTERISTICS AND DIFFICULTIES

\section{B. Model Definition}

In this paper, in the target monitoring area $\mathrm{W}$, the UAV network search process is abstracted as a network $\mathrm{G}$, which is defined as:

The network $G$ is divided into small squares, the monitoring range of each node is 27 squares, and the node is in the center of the cube.

UAV node makes relevant judgments of mobile according to the event that observed in the current monitoring range (27 square) and node.

Definition 1 (time factor): The node movement at current time is affected by the movement at the last moment.

Definition 2 (spatial factor): Nodes are affected by the surrounding nodes and events.

Definition 3 (geographical factor): The location of the node is affected by geographical restrictions, such as: UAV has a minimum flight height, etc.

Assumption 1: Do not consider the energy consumption, this paper only study the UAV node is how to move.

Assumption 2: The number of events and the number of nodes that have been visited in the history of the area are not known. In order to make the mobile model more close to the reality, it is difficult to make UAV record the history information in the practical application.

\section{3D MobiLity Model BASEd ON Visit DENSITy}

\section{A. Visit Density}

In the airborne network, the ability of real-time, fast and efficient monitoring and sensing of the target event determines the performance of a mobile model. The number of targets in a certain area, the number of nodes around, the mobile state of other nodes and other factors have a great impact on the performance of the mobile model.
Therefore, in this paper, we consider the 3D grid type network model, first, the number of events and the number of nodes around it are analyzed. Among them, according to a certain rule, the event is randomly generated in different squares, and the UAV can move in the network area according to the mobile model. For arbitrary square $d_{v}$, the event density $d_{e}$ is equal to the total number of events in the current time of the square, the UAV density $d_{u}$ is equal to the number of UAVs in the square at the current time.

This paper defines the visit density as:

$$
d_{v}=d_{e} /\left(1+d_{u}\right)
$$

The size of the visit density determine the needs of the current square to the UAV, when the number of events in the square is larger and the current number of UAVs is less, it shows that the square to the UAV's access to a larger demand.

\section{B. Density Change Product}

In practice applications, each event has independent lifetime, which would generate or die out randomly according to its own special laws. So if only consider the visit density of current space, regardless of the changeable trend of visit density, the events cannot be captured as more as possible. For example, the number of events in the region being monitored is num, and the number of events generated and died in unit time is num $_{\text {start }}$ and num end $_{\text {. So }}$ when num $_{\text {start }} /$ num $_{\text {end }}$ is greater than 1 , the number of events in the region will be gradually increasing over time. And the number will be decreasing if this ratio is smaller than 1 . We definite num $_{\text {start }} /$ num $_{\text {end }}$ as net generation rate of events in a unit area. Obviously, both visit density and net generation rate of events will affect the mobility of UAVs. And if the visit density and net generation rate is higher, the demand for UAVs is greater. Therefore, this issue definite DCP (Density Change Product) as:

$$
d c p_{v}=d_{v} * \text { num }_{\text {start }} / \text { num }_{\text {end }}
$$

where $d_{v}$ indicates the visit density of area $v$.

\section{3D-DGMM}

3D-GMM extends the 2D Gauss-Markov Model to three dimensions, and the velocity vector is computed as:

$$
\begin{aligned}
& s_{n}=\alpha s_{n-1}+(1-\alpha) \bar{s}+\sqrt{\left(1-\alpha^{2}\right)} s_{x_{n-1}} \\
& \theta_{n}=\alpha \theta_{n-1}+(1-\alpha) \bar{\theta}+\sqrt{\left(1-\alpha^{2}\right)} \theta_{x_{n-1}} \\
& p_{n}=\alpha p_{n-1}+(1-\alpha) \bar{p}+\sqrt{\left(1-\alpha^{2}\right)} p_{x_{n-1}}
\end{aligned}
$$


where $\alpha$ is the tuning parameter, $\bar{s}, \bar{\theta}$ and $\bar{p}$ are the mean speed and direction parameters, in which $\bar{\theta}$ is the mean horizontal pitch while $\bar{p}$ is the vertical. $s_{x_{n-1}}, \theta_{x_{n-1}}$ and $p_{x_{n-1}}$ are random variables from a Gaussian (normal) distribution that give some randomness to the new speed and direction parameters.

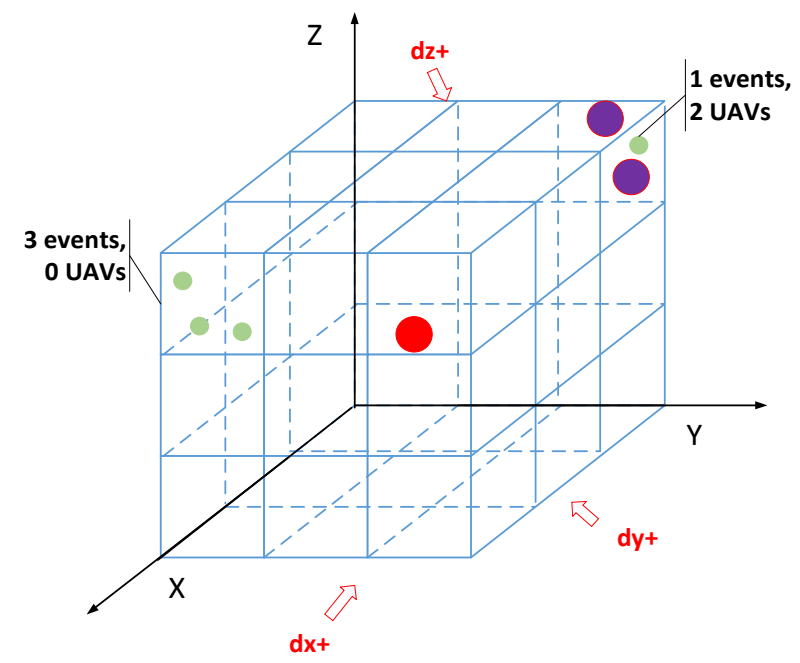

FIGURE II. MONITOR AREA OF UAV

In 3D-GMM model, the nodes move only according to definition of mobile, without considering other factors which may impact on the mobility of nodes, such as environment, time. Therefore, this article improve the Gaussian moving process based on the awareness and capture of events. The factors of visit density and changeable trend are brought into the Gaussian model, which makes the mobility of nodes has both the characters of Gaussian and event trend. The improved velocity vector is computed as:

$$
\begin{gathered}
s_{n}=\alpha s_{n-1}+(1-\alpha) \bar{s}+\sqrt{\left(1-\alpha^{2}\right)}(-1)^{\beta_{s}}\left|s_{x_{n-1}, \gamma_{s}}\right| \\
\theta_{n}=\alpha \theta_{n-1}+(1-\alpha) \bar{\theta}+\sqrt{\left(1-\alpha^{2}\right)}(-1)^{\beta_{\theta}}\left|\theta_{x_{n-1}, \gamma_{\theta}}\right| \\
p_{n}=\alpha p_{n-1}+(1-\alpha) \bar{p}+\sqrt{\left(1-\alpha^{2}\right)}(-1)^{\beta_{p}}\left|p_{x_{n-1}, \gamma_{p}}\right| \\
\beta_{s, \theta, p}=\left\{\begin{array}{l}
0, d c p^{+}>d c p^{-} \\
1, d c p^{+}<d c p^{-}
\end{array}\right. \\
\gamma_{s, \theta, p}=\left\lfloor\begin{array}{l}
\max \left(d c p_{s, \theta, p}\right) \\
\min \left(d c p_{s, \theta, p}\right)
\end{array}\right.
\end{gathered}
$$

where $\beta_{s, \theta, p}$ and $\gamma_{s, \theta, p}$ are the parameters in $s, \theta$ and $p$ three dimensions. $\beta_{s, \theta, p}$ will directly influence the direction of the
Gaussian movement with probability 1 , while $\gamma_{s, \theta, p}$ impact on the probability of the distance and direction to move. $\lfloor$. indicates rounding down and $\log ($.$) is used to determine the$ magnitude of two DCPs. $d c p^{+}$and $d c p^{-}$represent the sum of DCPs in opposite direction in three dimensional coordinate, in which $d c p^{+}$means the positive direction while $d c p^{-}$indicates the negative direction. As shown in Figure II, $d c p^{+}$is the sum of DCP in the nine square marked with red arrow.

Calculate the visit density and the generated and disappear number of events in unit time of all the 27 squares. Then according to Equations (2), get DCP of each square.

Compare the DCP of current square and the other 26 square. If the DCP in current square is greater than the others, the UAV has no need to move and should suspend to capture more events.

According to Equations (5), calculate $\beta_{s, \theta, p}$. If the sum of DCPs in font of UAV is larger, $\beta_{s, \theta, p}$ gets the value 0 . In opposite situation, it gets the value 1 .

According to Equations (6), calculate ${ }^{\gamma_{s, \theta, p}}$. The greater the gap between the DCPs in opposite direction is, the greater $\gamma_{s, \theta, p}$ is. If $\gamma_{s, \theta, p}$ is zero, it means the mobility model following Equations (4) is the same as which following Equations (3) and the mean of Gaussian distribution is zero. If $\gamma_{s, \theta, p}$ is not zero, the mean of Gaussian distribution is $\gamma_{s, \theta, p}$, which will increase the probability of moving a larger distance.

Finally, the UAV moves according to Equations (4).

\section{SimUlations}

In the simulation experiments for the research of UAV network, the experimental area is set to three-dimensional in which the length, width and height are $100 \mathrm{~km}$ respectively, and the side length of each square is $100 \mathrm{~m}$, the average speed of the node uniform random variable between $50 \mathrm{~m} / \mathrm{s}$ to $100 \mathrm{~m} / \mathrm{s}$. Nodes update their mobile status every $1 \mathrm{~s}$ time interval.

\section{A. The Mobility Track}

Figure III shows the track of 3D-DGMM under different parameters. It can be found from Figure III, as the parameter increases, the randomness of the node mobility track is reducing and the predictability is increasing. According to the literatures, the track of mobility model requires to be smooth, then track could be close to the practical. As shown in Figure III, when the parameters get the value 0.75 , the mobility track is smooth, and close to the practical movement of the UAV node, so we set the parameter to be 0.75 . 

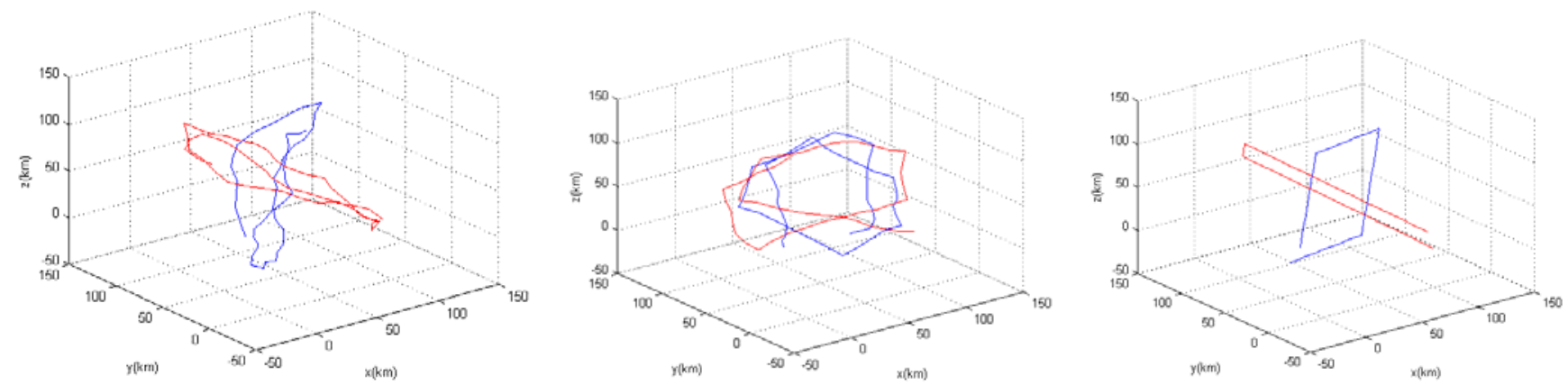

FIGURE III. MOBILITY TRACKS UNDER DIFFERENT PARAMETERS: (A) $\alpha=0.25$ (B) $\alpha=0.75$ (C) $\alpha=1$

\section{B. Correlation between Node Track and Events}

Figure IV shows the track generated in a period by 3DDGMM model and the positions of random events during this period. We can see that the track of the node is always surround by random events, which means that this mobility model is sensitive to events, and no sharp turns and other phenomena appear in the process of moving. The track is smooth and closes to the practical needs.
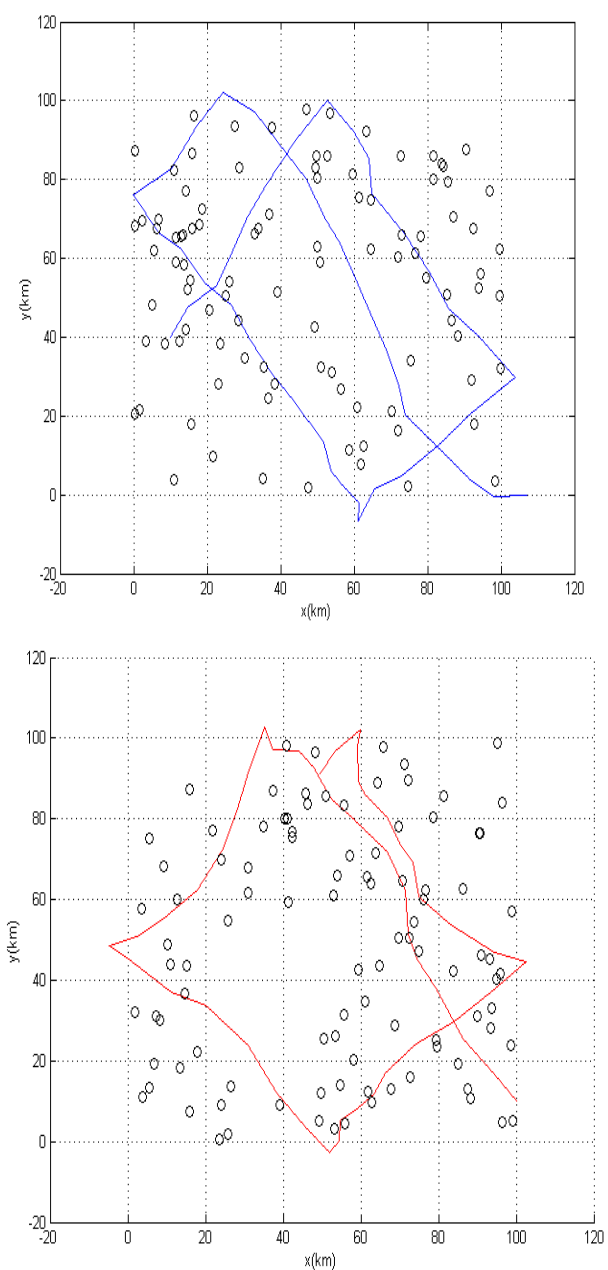

FIGURE IV. CORRELATION BETWEEN TRACK AND EVENTS
C. Comparison with 3D-GMM

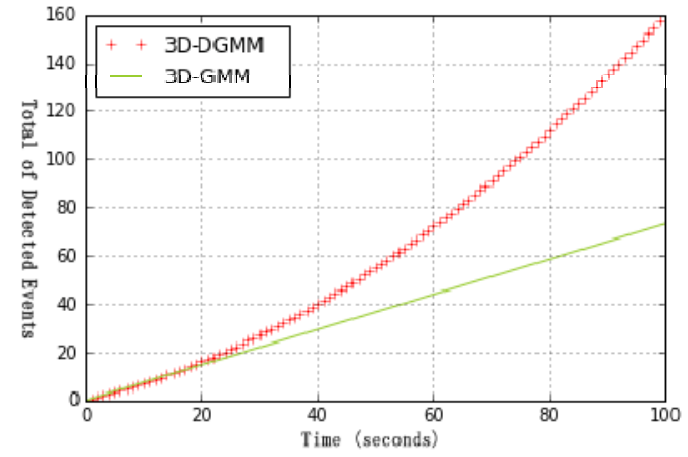

FIGURE V. COMPARISON WITH 3D-GMM

Figure $\mathrm{V}$ shows a comparison between the track for searching events generated by the 3D-DGMM mobile model and the general Gauss model. The X-axis is the time and Yaxis indicates total number of events searched. It can be seen that the curve of the two models is the beginning of the growth rate is closer. As time increases, the growth rate of red curve represented the 3D-DGMM mobile model is gradually larger than the green curve on behalf of the general Gauss mobility model. This is because in the general Gauss model, the construction of the mobile model does not take into account the impact of events and UAV on the mobile model. So it is not sensitive to the discovery of events. As the next position is randomly generated, only with the location of a node, and the formation and extinction of the event is also random, so the probability of explored events is the same, the slope remains unchanged. In this paper, our model is sensitivity of the events, so with the growth of time, the node in this model is moving forward to the direction of the events. So the growth rate for exploring events increased.

\section{CONCLUSIONS}

To build a reliable and stable UAV network, this paper analyzes the advantages and disadvantages of the existing UAV mobility models. On this basis, 3D-GMM model is improved, and a new three-dimensional Gauss Markova mobility model based on density change product (3D-DGMM) is proposed. First, the parameters are adjusted to ensure that the track of the node is smoother and practical. Then, density change product is brought in, which could control the next 
selection of the UAV mobility status to capture and sensing the target events better to maximize the coverage of the event. The results of simulation show the validity and rationality of the model, providing supports to evaluating the performance of three-dimensional environment UAV network. Next, it will conduct a detailed research on the communication of UAV network based on 3D-DGMM.

\section{ACKNOWLEDGMENT}

This work is supported by the National Basic Research Program of China (973 Program) under Grant No.2012CB315800, the National Natural Science Foundation of China under Grant No.61402521, No.61070173 and No.61103225, Jiangsu Province Natural Science Foundation of China under Grant No. BK20140068, Prospective Research Project on Future Networks of Jiangsu Future Networks Innovation Institute under Grant No.BY2013095-5-03.

\section{REFERENCES}

[1] Justin P R. Abdul J,Egemen K C . High-Dynamic Cross-layered aeronautical network architecture[J]. IEEE Transaction On Aerospace and Electronic Systems Vol.47,No.4 October 2011.

[2] M.Erturk, J.Haque, and H.Arslan.Challenges of Aeronautical Data Networks[C].Proc.IEEE Aerospace Conf.,Montana, Mar.2010, pp.1-7.

[3] Bekmezci I, Sahingoz O K, Temel Ş. Flying ad-hoc networks (FANETs): A survey [J]. Ad Hoc Networks, 2013, 11(3): 1254-1270.

[4] Xie J, Wan Y, Kim J H, et al. A survey and analysis of mobility models for airborne networks[J]. Communications Surveys \& Tutorials, IEEE, 2014, 16(3): 1221-1238.

[5] Broyles D, Jabbar A, and Sterbenz D. Design and Analysis of a 3-D Gauss Markov Mobility Model for Highly-dynamic Airborne Networks[C]. International Telemetering Conference, Las Vegas. NV,October 2009.

[6] Rohrer, J. P. AeroRP Performance in Highly-Dynamic Airborne Networks using 3D Gauss-Markov Mobility Model. MILCOM 2011 Military Communications Conference, ISSN 2155-7578, 2011, ISBN 9781467300797, pp. $834-841$

[7] Zhao M, Wang W. WSN03-4: A Novel Semi-Markov Smooth Mobility Model for Mobile Ad Hoc Networks[C]//Global Telecommunications Conference, 2006. GLOBECOM'06. IEEE. IEEE, 2006: 1-5.

[8] Zheng B, Zhang H Y, Huang G C, et al. Design and implementation of a 3-D smooth mobility model[J].Journal of Xidian University, 2011, 38(6): 179-184.

[9] He M, Chen Q L, Chen X L, et al. Fish swarm inspired Ad hoc networks node random mobility optimization model in $3 \mathrm{D}$ environment $[\mathrm{J}]$. ChineseJournal of Scientific Instrument, 2014, 35(12): 2826-2834 